\title{
Cluster based Event Driven Routing Protocol (CERP) for Wireless Sensor Network
}

\author{
Sohini Roy \\ Calcutta Institute of Engineering and Management \\ 24/A Chandi Ghosh Road, Kolkata 700040
}

\author{
Ayan Kumar Das \\ Calcutta Institute of Engineering and Management \\ 24/A Chandi Ghosh Road, Kolkata 700040
}

\begin{abstract}
Energy efficiency is the main challenge in wireless sensor network. Many routing protocols have been designed to face this challenge. Hierarchical cluster-based routing is one of the efficient techniques to route the sensed data from source node to sink node. The proposed algorithm deals with cluster formation based on different events, cluster head selection, aggregation of the sensed data within a cluster and sending that to the base station. Simulation result proves that the performance of the proposed scheme is better than Energy Efficient Clustering Algorithm for data aggregation in WSNs (EECA) and A Multipath Routing Protocol Based on Clustering and Ant Colony Optimization (MRP) for Wireless Sensor Networks.
\end{abstract}

\section{General Terms}

Energy Efficient Routing, Event driven cluster formation, Cluster Head selection

\section{Keywords}

Wireless Sensor Network, Cluster Head, Heterogeneous, Network Lifetime, Energy Efficiency, Data aggregation

\section{INTRODUCTION}

A wireless sensor network is composed of many sensor nodes, which are densely deployed in a remote area. The sensor nodes are static in nature and are solely devoted to the task of sensing data from the environment and send that to the base station. The flat routing protocols like SPIN [1], DD [2], MERCC [3], MESR [4], A Novel Power-Balancing Routing Scheme for WSN [5], consume much energy in sending the sensed data to the base station. The nodes of the sensor network are equipped with limited power, low computational ability and less memory. This is the reason why such a routing algorithm should be energy efficient as well as should have low computational cost. Cluster-based routing such as LEACH [6], HEED [7], EECS[8] etc. serves this purpose where a head node, also called the cluster head, gathers data from its cluster members, aggregate that and sends that to the base station. This helps to save energy by sending the data through cluster head instead of sending from each individual node, which also reduces redundant data, controls the congestion of the network and increases network lifetime.

The common hierarchical cluster-based routing protocols like LEACH [6] include direct sending of the aggregated data from the cluster head of each cluster to the base station. In cases of larger networks this scheme is not always feasible. Thus, CERP follows a multi-hop path to send the data to the base station. In this algorithm clusters are formed on the basis of the occurrence of events and the cluster head selection process is based on certain parameters like remaining energy, number of member nodes and distance between them. This is followed by sensing of data, data aggregation by the selected cluster head and sending of data to the base station following the shortest path which is determined by Dijkstra's algorithm [9]. The role of cluster head is rotated between the nodes of each cluster in every round for even distribution of energy.

\section{RELATED WORKS}

The clustering technique adopted by different algorithms has certain advantages and disadvantages. It is also not possible to adopt all the advantageous options in a particular algorithm, thus various routing protocols are developed to meet the challenges at the optimum level.

Energy Efficient Heterogeneous Clustered scheme for Wireless Sensor Network [10] enhances the performance by the introduction of heterogeneity and by the selection of cluster heads based on certain parameters like average distance of a cluster head from its member nodes, average distance of the cluster head from the base station and the transmission range of the amplifier. Performance comparison of LEACH and LEACH-C protocols using NS2 [11] mentions that the distributed clustering includes identity based clustering as in LCA [12] where an unique id identifies a node and the node with the highest id is chosen as the cluster head, neighborhood information based clustering as in HCCA [13] in which a node having the highest number of neighbors is selected as cluster head, probabilistic clustering as in LEACH [6] where the role of cluster head is rotated among the sensor nodes for balancing energy, iterative clustering as in DCA [14] or SPAN [15] where delayed willingness announcement technique is applied for choosing a node as cluster head on the basis of weight. The centralized approach, on the other hand includes protocols such as LEACH-C [16] which proposes the sending of location awareness of the nodes to the base station, which in turn would select the cluster head on the basis of remaining energy. PEGASIS [17] includes arranging of nodes into chains so that they can communicate only with their closest neighbors, thereby minimizing the power requirement for data transmission per second and reducing overhead. However, failure of any intermediate node can cut off the link between other nodes and thus can detach a portion of the network. In HEED [7] the cluster head is selected on the basis of residual energy of nodes which leads to proper energy dissipation. Although ignoring the network structure brings about uneven energy consumption throughout the network. Optimal Energy Allocation in heterogeneous Wireless Sensor Network [18] mentions about sensors having different sensing range and different battery capacities. Nodes near the base station are equipped with more energy. Deployment of nodes is done in an arbitrary topology in an area such that they are connected. The paper finds optimal initial energy allocation to the sensors for prolonging the network lifetime. The expected number of working sensors at each time and the data 
transmission rate are also calculated. Energy Efficient Clustering Algorithm for data aggregation in WSN [19] states two phases of clustering. One is the formation of clusters, which includes the broadcasting of messages by each node to the neighbor nodes, advertising the radius, residual energy and co-ordinates. This is followed by calculation of competition bids and selection of a node as cluster head, which in turn broadcasts the message that it is the head. The other phase is Data Aggregation and tree construction which include calculation of weight on the basis of distance from the base station and then broadcasting of that weight. A Multipath Routing Protocol Based on Clustering and Ant Colony Optimization for Wireless Sensor Networks [20] is based on Multipath Routing Protocol (MRP). In this algorithm, the formation of the cluster is event driven and the node lying at the center of the event is chosen as the cluster head. The cluster head divides the load after aggregation into smaller parts and selects multiple paths to the base station from it using Ant Colony Optimization to send its data.

\section{PROPOSED WORK}

\subsection{Basic Concept}

The proposed scheme assumes the nodes of the network to be static, the base station or sink node is of high configuration, that contains high energy, enough memory and is deployed in a controllable place outside the network region. It is also assumed that all sensor nodes are capable of data aggregation, computing their own residual energy and finding their geographical location. The energy consumption of each node for transmitting or receiving a packet depends upon the distance between the transmitter and the receiver and the size of the packet.

CERP can be divided into four phases: The cluster formation, cluster head selection, the sensing and data aggregation phase and the phase of sending the sensed data to the base station.

In WSN sensor nodes are deployed very densely. Thus, many nodes of a small region can sense the same event and might want to send that redundant data through multiple paths, which leads to wastage of energy as well as congestion in the network. This can be solved by grouping the nodes in a nearby location, called cluster and selecting a head node, called the cluster head, among those nodes in that location. All the nodes will send the sensed data to its cluster head; the cluster head will aggregate this data, eliminate the redundant data and transmit that to the sink node. Most of the existing algorithms do not check the occurrence of events at the time of cluster formation phase. This results in the formation of clusters without the occurrence of any event, thus wasting energy. It may also happen that nodes that sense a single event belong to different clusters and therefore more than one cluster head have to get involved in sending the same data to the base station. To avoid these drawbacks, CERP has adopted an event-based clustering scheme, in which, formation of clusters is initiated by the occurrence of an event in the network region.

Cluster head selection is the next phase. In each round, the nodes of a cluster compete to become the cluster head. If the residual energy $\left(\mathrm{E}_{\mathrm{Ri}}\right)$ of node $\mathrm{i}$ is greater than the threshold value $(\mathrm{E})$ then it becomes a candidate node of the cluster head selection process. The threshold value can be defined as the minimum energy required in receiving data from all nodes, aggregating them, and sending that to a neighbor node. Every candidate node will calculate the competition bid $\left(\mathrm{CV}_{\mathrm{i}}\right)$ value for being selected as a cluster head.

$$
\mathrm{CV}_{\mathrm{i}}=\mathrm{E}_{\mathrm{Ri}} * \mathrm{~N}_{\mathrm{adj}}
$$

Where, $\mathrm{N}_{\mathrm{adj}}$ is the number of adjacent nodes of node $\mathrm{i}$.

After the competition bid calculation, each node of a cluster broadcasts its own $\mathrm{CV}_{\mathrm{i}}$ value to all its neighbor nodes. Each node receives the $\mathrm{CV}_{\mathrm{i}}$ value of all its neighbors and compares them with its own $\mathrm{CV}_{\mathrm{i}}$ value and finally the node with the highest $\mathrm{CV}_{\mathrm{i}}$ value is chosen as the cluster head. The energy $\left(\mathrm{E}_{\mathrm{Bdi}}\right)$ consumed by each node for broadcasting the $\mathrm{CV}_{\mathrm{i}}$ value is:

$$
\mathrm{E}_{\mathrm{Bdi}}=\mathrm{k} * \sum_{\mathrm{j}=1}^{\mathrm{n}} \mathrm{d}_{\mathrm{ij}} * \mathrm{sz}
$$

Where $\mathrm{n}$ is the number of nodes in the cluster, sz is size of the message, $d_{i j}$ is the distance between node $i$ and its neighbor $j$ and $\mathrm{k}$ is the constant of proportionality. The value of $\mathrm{k}$ is determined by trial and error method. The same energy is consumed by each node in receiving the $\mathrm{CV}_{\mathrm{i}}$ values which is denoted as $E_{R d i}$. Thus remaining energy $\left(E_{R i}\right)$ of each node after broadcasting the $\mathrm{CV}_{\mathrm{i}}$ is:

$$
E_{R i}=E_{\text {initial }}-\left(E_{B d i}+E_{R d i}\right)
$$

The distance between any pair of nodes $\mathrm{i}$ and $\mathrm{j}$ is calculated using:

$$
d_{i j}=\sqrt{\left[\left(x_{j}-x_{i}\right)^{2}+\left(y_{j}-y_{i}\right)^{2}\right]}
$$

The node containing the maximum $\mathrm{CV}_{\mathrm{i}}$ value will send the success message to all its neighbors and the energy consumed in declaring the success message by the node having the maximum value of $\mathrm{CV}_{\mathrm{i}}$ is:

$$
\mathrm{E}_{\mathrm{dc}}=\mathrm{k} * \sum_{\mathrm{i}=1}^{\mathrm{n}}\left(\mathrm{d}_{\mathrm{ic}} * \mathrm{sz}\right)
$$

Where, $\mathrm{n}$ is the number of member nodes, $\mathrm{d}_{\mathrm{ic}}$ is the distance between each member node and the selected cluster head node, sz is the size of the declaration message. The energy consumed by each member node for receiving the declaration message is:

$$
\mathrm{E}_{\mathrm{dm}}=\mathrm{k} *\left(\mathrm{~d}_{\mathrm{ic}} * \mathrm{sz}\right)
$$

After broadcasting the declaration message all the nodes of a cluster are updated about the cluster head of the current round and thus the phase of cluster head selection completes.

The next phase comprises of data sensing and aggregation. As each node of a cluster gets to know which node is the cluster head of the current round, the nodes that are within the sensing radius of the cluster head start the sensing phase. The energy lost by each node due to sensing of data is given as:

$$
\mathrm{E}_{\mathrm{S}}=\mathrm{k} * \mathrm{~S}_{\mathrm{i}} * \mathrm{t}
$$

Where, $\mathrm{k}$ is a constant, $\mathrm{S}_{\mathrm{i}}$ is size of the data sensed by node $\mathrm{i}$ and $t$ is the time taken for sensing the data. After sensing, each member node forwards the sensed data to the cluster head and the energy required to send data to the cluster head is:

$$
\mathrm{E}_{\mathrm{TS}}=\mathrm{k} *\left(\mathrm{~d}_{\mathrm{ic}} * \mathrm{~S}_{\mathrm{i}}\right)
$$

Where $d_{i c}$ is the distance between the node $i$ and the cluster head. The energy consumed by the cluster head in receiving the sensed data from the member nodes is given by: 


$$
\mathrm{E}_{\mathrm{RC}}=\mathrm{k} * \sum_{\mathrm{i}=1}^{\mathrm{n}}\left(\mathrm{d}_{\mathrm{ic}} * \mathrm{~S}_{\mathrm{i}}\right)
$$

After receiving the sensed data from the member nodes, the cluster head removes the redundant data, aggregates the data and compress them. The energy consumed by the cluster head for aggregating and compressing the received data is given by:

$$
\mathrm{E}_{\mathrm{a}}=\mathrm{k} * \sum_{\mathrm{i}=1}^{\mathrm{n}} \mathrm{S}_{\mathrm{i}} * \mathrm{n}
$$

Where, $S_{i}$ is size of data received from each node, $n$ is number of active member nodes sending data.

Next starts the phase of sending the aggregated data to the base station. After selection of the cluster head the aggregated data should reach to the base station in an energy efficient way. CERP follows the shortest route to the base station from a cluster head for sending the aggregated data to the base station, so that the base station can get the information about the event in a very short time and can get a chance to take the corrective actions. The shortest route from the cluster head node of a cluster to the base station may consist of entirely non-cluster head nodes. This is done to avoid the quick depletion of energy of the cluster head nodes. To find the shortest route to the base station Dijkstra's shortest path finding algorithm is used.

The Dijkstra's algorithm [9] finds the shortest route to a destination based on weight from a single source. The distance between the nodes is considered as the weight value in CERP. The cluster head sends the aggregated data to the sink node through the shortest path.

The energy consumed by the sender cluster head node for sending its aggregated data to the closest neighbor node $i$ is given as:

$$
\mathrm{E}_{\mathrm{Ci}}=\mathrm{k} *\left(\mathrm{~d}_{\mathrm{ic}} * \mathrm{sz}\right)
$$

Where $d_{c i}$ is the distance between the cluster head node and its closest neighbor $\mathrm{i}, \mathrm{sz}=$ the size of the aggregated data.

The same energy is consumed in receiving the packet by the next-hop node $\mathrm{i}$. The node to which the cluster head forwards the aggregated data, again forwards it to the next node in the shortest path and so on. The energy consumed by each node in the shortest path to transmit the aggregated data is:

$$
\mathrm{E}_{\mathrm{F}}=\mathrm{k} *\left(\mathrm{~d}_{\mathrm{ij}} * \mathrm{sz}\right)
$$

The same energy is used up by each node in receiving the aggregated data from the adjacent sender node. The nodes that are within the 2-hop distance from the base station can directly forward the aggregated data to the base station if the weight of the link between that node and the base station is the minimum. The energy required by a node, close to the base station, to forward the data to the base station is given as:

$$
\mathrm{E}_{\mathrm{FB}}=\mathrm{k} *\left(\mathrm{~d}_{\mathrm{Bi}} * \mathrm{sz}\right)
$$

Where $d_{B i}$ is the distance of the node $i$ from the base station. The total energy consumption of the nodes constituting the shortest route through which data is sent to the base station from a cluster head to the base station is given as:

$$
\begin{aligned}
E(p)=\sum_{\mathrm{k}=1}^{\mathrm{m}-1}\left(\mathrm{E}_{\mathrm{t}}+\mathrm{E}_{\mathrm{cpu}}+\mathrm{E}_{\mathrm{r}}\right) & \\
& =\left(\mathrm{E}_{\mathrm{t}}+\mathrm{E}_{\mathrm{cpu}}+\mathrm{E}_{\mathrm{r}}\right)(\mathrm{m}-1)
\end{aligned}
$$

Where $\mathrm{E}(\mathrm{p})$ is the total energy consumed through path $\mathrm{p}, \mathrm{E}_{\mathrm{t}}$ is energy required to transmit data, $E_{c p u}$ is the energy required for computation, $\mathrm{E}_{\mathrm{r}}$ is the energy required to receive data and $\mathrm{m}$ is the number of nodes constituting the path including the sender cluster head node.

The role of cluster head is rotated among the nodes of a cluster. Thus, if the cluster remains same for more than one round, different shortest route is selected from the different cluster heads by Dijkstra's algorithm. This avoids the selection of the same nodes in the shortest path again and again, thus provides uniform distribution of energy.

\subsection{Case Study}

A network of 25 nodes is considered. Fig. 1 shows the cluster formation phase of CERP. The two events occurring in the network at a particular round are sensed by node number 9 and 22. These two nodes as the initiator nodes broadcast the request message to the neighbors at one hop distance from them and those nodes also forward that to nodes at one hop distance from them.

Clusters are formed by the initiator nodes and the nodes receiving the request messages from the initiator nodes. Only the nodes that are included in a cluster will take part in sensing the events.

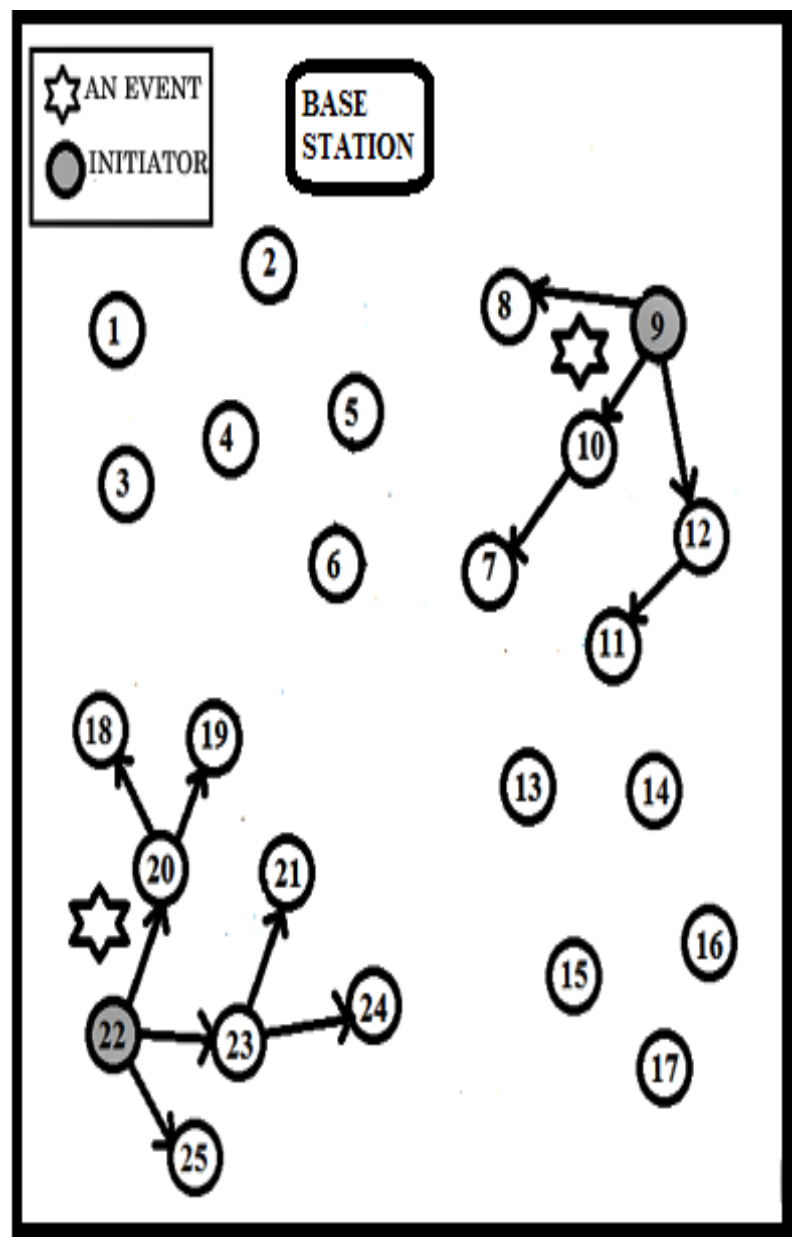

Fig 1: Initiator node broadcasting request message thereby initiating cluster formation

In Fig. 2 node number 10 and node number 20 are selected as cluster heads of the current round based on the $\mathrm{CV}_{\mathrm{i}}$ value. The nodes that are not in any cluster will only take part in sending the data to the base station. 
While sending the aggregated data from each cluster head node to the base station, the shortest route is followed using Dijkstra's algorithm. The shortest path is selected on the basis of distance (dt) between the nodes. Node 20 is the cluster head of the second cluster, which follows the path 20-19-6-5-2Base_Station to send the data.

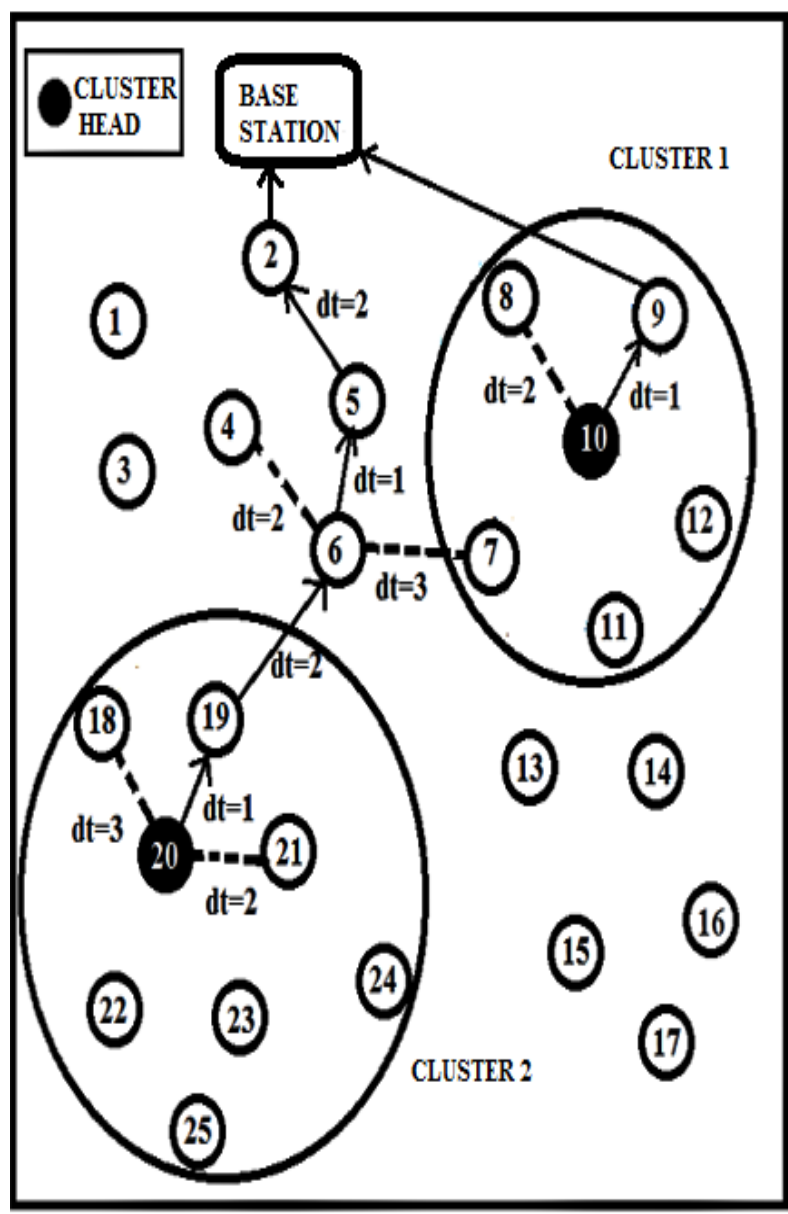

Fig 2: Data transmission to base station following Dijkstra's Algorithm

\subsection{Algorithm}

The algorithm is divided into four modules namely: Cluster_formation(), CH_selection(), Sensing_Aggregation() and Send().

\subsubsection{Initialization \\ STEP 1: Begin}

STEP 2: Each node gets their $\mathrm{x}$ and y co-ordinate value.

STEP 3: Distance between every pair of nodes and the distance from the base station is calculated by n nodes.

STEP 4: If no event occurs then

Nodes remain idle.

STEP 5: Else

- Cluster_formation() function is called by the initiator node which first senses the event

- CH_selection() function is called by initiator of each cluster.

- Sensing_Aggregation() function is called by the cluster head of each cluster.
- Send() function is called by the cluster head of each cluster.

STEP 6: End

\subsubsection{Function Cluster_formation()}

// forms clusters on the occurrence of an event.

\section{STEP 1: Begin}

STEP 2: Initiator node broadcasts request message to the nodes at one hop distance.

STEP 3: Those nodes send back an acknowledgement message to the initiator and forward the request to the nodes at one hop distance to them.

STEP 4: Nodes within 2-hop distance from the initiator join the initiator to form a cluster.

STEP 5: Return

\subsubsection{Function CH_selection() \\ // selects the cluster head of each round.}

\section{STEP 1: Begin}

STEP 2: The residual energy $E_{R i}$ is calculated by each node in the cluster.

STEP 3: If $E_{R i}>$ Threshold Energy then---

- $\mathrm{CV}_{\mathrm{i}}$ (the competition bid for becoming a cluster head) of each node in a cluster is calculated.

- $\quad$ Set $\mathrm{CV}_{\mathrm{i}}=\mathrm{k} * \mathrm{E}_{\mathrm{Ri}} * \mathrm{~N}_{\mathrm{adj}}$

STEP 4: The node with the maximum value of $\mathrm{CV}_{\mathrm{i}}$ is found and that node is selected as the cluster head node for the current round of that cluster.

STEP 5: The cluster head node broadcasts the success message to the neighbor nodes.

STEP 6: Return

\subsubsection{Function Sensing_Aggregation()}

// sensing of data and data aggregation is done.

STEP 1: Begin

STEP 2: If node i belongs to a cluster

- Start sensing of data

- Send the sensed data to the cluster head

Else

- Remain idle.

STEP 3: Aggregate the sensed data received by the cluster head.

STEP 4: Return

\subsubsection{Function Send()}

// sends the aggregated data to the Base station

\section{STEP 1: Begin}

STEP 2: Find the shortest route to the Base station using Dijkstra's algorithm.

STEP 3: Send the aggregated data to the Base station through the shortest path.

STEP 4: Return 


\section{SIMULATION RESULT}

To analyze the performance of the algorithm, a network with 50 nodes is created and it is considered that 20 events are taking place within 20 rounds.

A round can be considered as a total time to form a cluster, followed by cluster head selection, data sensing, aggregation and sending the aggregated data to the base station. The simulation is performed on MATLAB platform considering a minimum of 20 rounds.

The parameter list is given below-

Table 1. Parameter List

\begin{tabular}{|l|l|}
\hline Parameters & Description \\
\hline Network size & 50 nodes \\
\hline Initial energy & 50J per node \\
\hline MAC Protocol & IEEE 802.15 .4 \\
\hline Power consumption & $\begin{array}{l}\text { Equivalent to packet size } \\
\text { and distance }\end{array}$ \\
\hline Number of rounds & At least 20 \\
\hline
\end{tabular}

The initial battery power of every node is considered 50 Joules. The size of each packet of data is taken as 10 Bytes. After some rounds the total reduction in energy of the network and the total number of dead nodes is calculated. The result obtained is compared with that of Energy Efficient Clustering Algorithm (EECA) [19] and A Multipath Routing Protocol Based on Clustering and Ant Colony Optimization (MRP) for Wireless Sensor Networks [20], which proves that after the 20th round more nodes are dead for both EECA and MRP with compare to CERP.

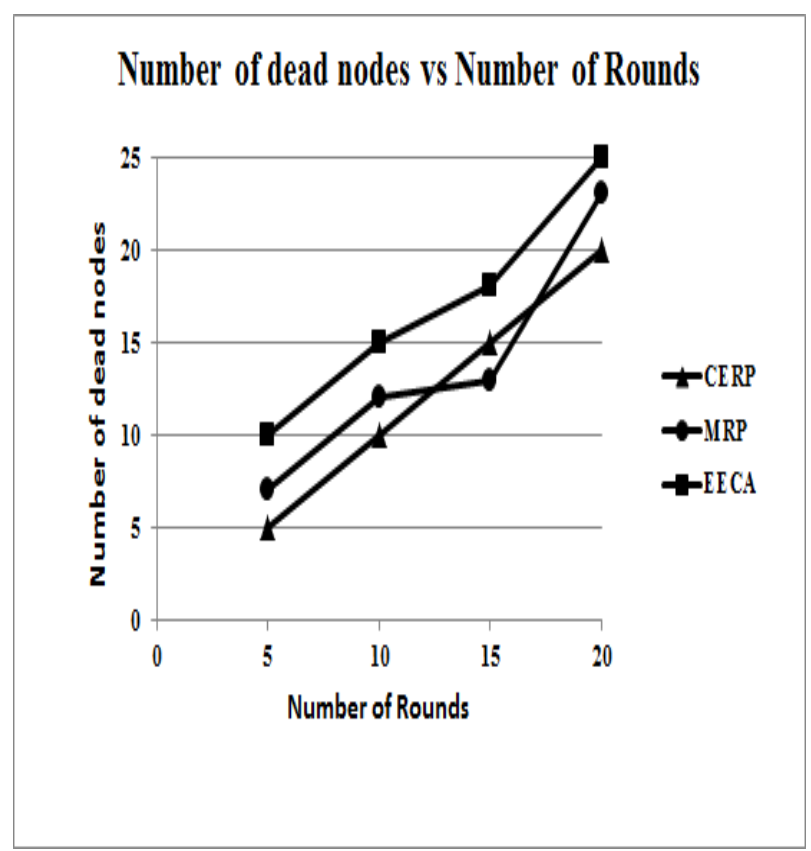

Fig 3: Number of dead nodes vs. Number of rounds
Again, after each round the average residual energy of the network is measured for all the three algorithms and has been plotted.

Fig. 4 shows that after 20th round the algorithm EECA [19] and MRP [20] used up more energy than CERP. It can also be seen from the graph in Fig. 4 that the slope of the curve showing the decrement in average residual energy with the increase in the number of rounds is lesser in case of CERP than that of EECA and MRP. So, it can be concluded that the average residual energy decreases at a slower rate in CERP. Thus the proposed algorithm CERP can send the aggregated data to the base station in an energy efficient way and increases network lifetime.

\section{Average residual energy vs Number of Rounds}

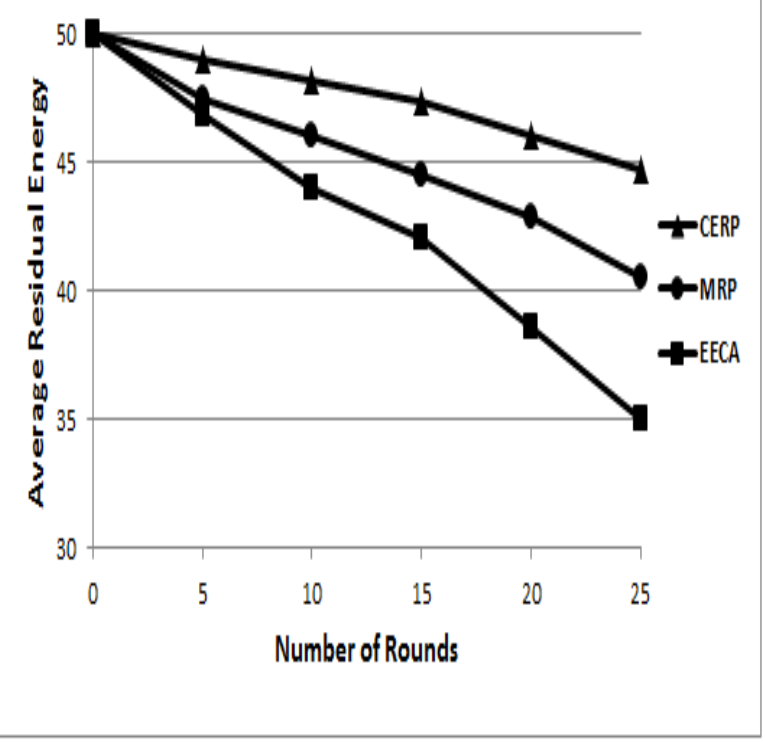

Fig 4: Average residual energy vs. Number of rounds

\section{CONCLUSION}

Most of the existing routing protocols for wireless sensor network are making clusters in order to reduce the energy consumption, regardless of the occurrence of events. If no event is there, cluster formation makes no meaning except energy wasting. The proposed protocol CERP makes clusters only if there are events. The simulation result shows that the performance is better than other algorithms like EECA [19] and MRP [20], as in this algorithm average residual energy of the network is not going down very quickly, thus increasing the network lifetime.

\section{REFERENCES}

[1] J. Kulik, W.R. Heinzelman and H. Balakrishnan, "Negotiation based protocols for disseminating information in wireless sensor networks.", Wirel. Netw.Vol.8, pp. 169-185, 2002.

[2] C. Intanagonwiwat, R. Govindan, D. Estrin and J. Heidemann, "Directed diffusion for wireless sensor networking", IEEE/ACM Trans. Netw. Vol.11, pp.2-16, 2003.

[3] Ayan Kumar Das and Dr. Rituparna Chaki, "MERCC: Multiple Events Routing with Congestion Control for WSN", ACITY (1), Vol. 176, pp. 691-698, 2012. 
[4] Ayan Kumar Das and Dr. Rituparna Chaki, "Multiple Event Source Routing (MESR) for Energy Efficient Wireless Sensor Network", International Journal of Computer Applications, Vol. 39, No 15, doi: 10.5120, pp.4894-7401, 2012.

[5] Ayan Kumar Das and Dr. Rituparna Chaki, "A Novel Power-Balancing Routing Scheme for WSN", Communications in Computer and Information Science, Springer, Vol. 162, 2011, pp 24-34.

[6] W.B. Heinzelman et al., An application-specific protocol architecture for wireless microsensor networks, IEEE Transactions on Wireless Communications 1 (4) (2002) 660-670.

[7] Harneet Kour and Ajay K. Sharma, "Hybrid Energy Efficient Distributed Protocol for Heterogeneous Wireless Sensor Network", Internatioanl Journal of Computer Applications, Vol.4(6), pp. 0975-8887, July 2010.

[8] M. Ye, C. Li, G. Chen and J. Wu, "EECS: An Energy Efficient Clustering Scheme in Wireless Sensor Networks", In Proceedings of the 24th IEEE International Performance, Computing, and Communications Conference (IPCCC), Phoenix, AZ, USA, 7-9; pp. 535-540, April 2005

[9] E.W. Dijkstra, "A Note on Two Problems in Connexion with Graphs", Numerische Mathematik 1, 269-271. (1959)

[10] Dilip Kumar, Trilok C. Aseri and R.B. Patel, "Energy Efficient Heterogeneous Clustered scheme for Wireless Sensor Network", Journal of Computer Communications, Elsevier, Vol. 32(4), pp. 662-667, doi: 10.1016/j.comcom.2008.11.025, March 2009.

[11] Geetha. V.a, Pranesh.V. Kallapurb and Sushma Tellajeerac, "Clustering in Wireless Sensor Networks: Performance Comparison of LEACH \& LEACH-C Protocols Using NS2", in Elsevier journal 2012.

[12] D.J. Baker and A.Epheremides, "The Architectural Organization of a Moblie Radio Network via a Distributed Algorithm",IEEE Transactionson Communications, vol.29, no. 11, November 1981.
[13] P. Kumarawadu, D. J. Dechene and M. Luccini, A. Sauer, "Algorithms for Node Clustering in Wireless Sensor: A Survey”, Proceedings of IEEE 2008.

[14] Abusayeed Saifullah, You $\mathrm{Xu}$, Chenyang $\mathrm{Lu}$ and Yixin Chen, "Distributed Channel Allocation Protocols for Wireless Sensor Networks", IEEE Transactions on Parallel and Distributed Systems, IEEE computer Society Digital Library, IEEE Computer Society, 29 July 2013.

[15] Benjie Chen, Kyle Jamieson, Hari Balakrishnan, and Robert Morris, "Span: An Energy Efficient Coordination Algorithm for Topology Maintenance in Ad Hoc Wireless Networks", ACM Wireless Networks Journal, Vol.8, 2002.

[16] Navneet Verma, Dr. Devendra Prasad and Mukesh Chawla, "Energy and Throughput of Hierarchical Routing Protocol Leach and Leach-C for WSNS: A Review", INTERNATIONAL JOURNAL OF RESEARCH IN MANAGEMENT \& TECHNOLOGY, Vol.1,No.1.

[17] Stephanie Lindsey, Cauli Raghavendra and Krishna M. Sivalingam, "Data Gathering Algorithm in Sensor Networks Using Energy Metrics", IEEE TRANSACTIONS ON PARALLEL AND DISTRIBUTED SYSTEMS, VOL.13, NO.9, SEPTEMBER 2002.

[18] Keqin Li and Jie Li, "Optimal energy allocation in heterogeneous wireless sensor networks for lifetime maximization", Journal of Parallel and Distributed Computing, Elsevier, Vol. 72(7), pp.902-916, July 2012.

[19] Chao SHA, Ru-chuan WANG, Hai-ping HUANG and Li juan SUN, "Energy efficient clustering algorithm for data aggregation in wireless sensor networks", Nanjing University of Posts and Telecommunications, Nanjing 210003, China, The Journal of China Universities of Posts and Telecommunications, January 2011.

[20] Jing Yang, Mai Xu, Wei Zhao and Bhaoguo Xu, " A Multipath Routing Protocol Based on Clustering and Ant Colony Optimization for Wireless Sensor Networks", Sensors 2010, Vol. 10(5),pp.4521-4540; doi: $10.3390 / \mathrm{s} 1005040521$ 